\title{
The Effect of Chair Type on Users' Viewing Experience for 360-degree Video
}

\author{
Yang Hong \\ Department of Computer Science \\ University College London \\ uczl134@ucl.ac.uk
}

\author{
Andrew MacQuarrie \\ Department of Computer Science \\ University College London \\ andrew.macquarrie.13@ucl.ac.uk
}

\author{
Anthony Steed \\ Department of Computer Science \\ University College London \\ a.steed@ucl.ac.uk
}

\begin{abstract}
The consumption of 360-degree videos with head-mounted displays (HMDs) is increasing rapidly. A large number of HMD users watch 360-degree videos at home, often on non-swivel seats; however videos are frequently designed to require the user to turn around. This work explores how the difference in users' chair type might influence their viewing experience. A between-subject experiment was conducted with 41 participants. Three chair conditions were used:fixed, half-swivel and full-swivel. A variety of measures were explored using eye-tracking, questionnaires, tasks and semistructured interviews. Results suggest that thefixed and half-swivel chairs discouraged exploration for certain videos compared with the full-swivel chair. Additionally, participants in thefixed chair had worse spatial awareness and greater concern about missing something for certain video than those in the full-swivel chair. No significant differences were found in terms of incidental memory, general engagement and simulator sickness among the three chair conditions. Furthermore, thematic analysis of post-experiment interviews revealed four themes regarding the restrictive chairs: physical discomfort, difficulty following moving objects, reduced orientation and guided attention. Based on thefindings, practical implications, limitations and future work are discussed.
\end{abstract}

\section{CCS CONCEPTS}

- Human-centered computing $\rightarrow$ Empirical studies in $\mathrm{HCI}$; Displays and imagers; Empirical studies in visualization;

\section{KEYWORDS}

Cinematic virtual reality, panoramic video, user study

\section{ACM Reference Format:}

Yang Hong, Andrew MacQuarrie, and Anthony Steed. 2018. The Effect of Chair Type on Users' Viewing Experience for 360-degree Video. In VRST 2018: 24th ACM Symposium on Virtual Reality Software and Technology (VRST '18), November 28-December 1, 2018, Tokyo, fapan. ACM, New York, NY, USA, 11 pages. https://doi.org/10.1145/3281505.3281519

\section{INTRODUCTION}

Over the past few years, head-mounted display (HMD)-based virtual reality has gained increasing popularity. A notable use of HMDs is

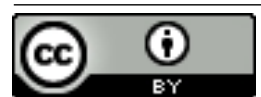

This work is licensed under a Creative Commons Attribution International 4.0 License. VRST '18, November 28-December 1, 2018, Tokyo, Japan

(C) 2018 Copyright held by the owner/author(s).

ACM ISBN 978-1-4503-6086-9/18/11.

https://doi.org/10.1145/3281505.3281519 the watching of panoramic videos. This offers a different, immersive experience compared to traditionalfilm and TV formats. In January 2017, Samsung reported thatfive million Gear VR HMDs were in use, and that consumers had viewed over 10 million hours of video on the device [6]. While more recentfigures have not yet been made available by manufacturers, VR intelligencefirm ARtillry estimated in March 2018 that the global install base for HMDs was 26 million [1]. Top apps on the Gear VR also suggested the popularity of video consumption in these devices [28].

Many factors could have an influence on viewing experience with HMDs, such asfield of view, resolution, etc. One aspect that may have an impact is the type of chair a user is sitting in. While a lot of public demonstrations of HMDs use swivel chairs, at home, users might sit on a chair that affords little movement. As noted in previous research [17], afixed chair for HMD viewing might place a soft limit on thefield of regard (FOR), thus discouraging users from exploring the content of the videos. This point would have important implications for the design of panoramic videos, as at-home HMD users are more likely to be seated on afixed couch. If the restriction of afixed chair on exploration is true, creators of panoramic videos need to design content intentionally to meet the preferences of at-home users who might not actively explore much of the video.

Further evidence of this limited 360-degree video exploration is emerging from industry. YouTube collects details on the viewing habits of users watching 360-degree media. This data allowed YouTube to conclude that "people spent $75 \%$ of their time within the front 90 degrees of a video" [30]. It is statistics such as this that have led YouTube to develop VR180, a format for immersive video in which a horizontalfield-of-view of only 180 degrees is available [31]. Similarly, the influential 360-degree media production company Jaunt suggest that "it's best to keep the action within 150-degrees" [14].

However, YouTube do not collect data on the physical situation of the user, such as the type of chair the viewer is using. This is an important consideration, as the physical set-up may play a role in the extent to which users explore content. An understanding of the relationship between the physical set-up and exploration is critical in order to correctly advise creators on how users might engage with their content in different scenarios. Therefore, this paper looks at whether there is an effect of chair type on users' HMD viewing experience for panoramic videos. 


\section{PREVIOUS WORKS}

\subsection{Panoramic Videos}

The production and consumption of panoramic videos is growing quickly due to the improvement of consumer and professional panoramic video cameras. The New York Times is creating one new panoramic video per day [18]. Over one million 360-degree videos have been uploaded to Facebook [2], with millions of users watching them every day [9]. 360-degree videos are also being viewed immersively, with Samsung stating in January 2017 that five million Gear VR HMDs were in use, and that consumers had watched over 10 million hours of video on the device [6]. VR intelligence firm ARtillry estimated in March 2018 that the global install base for HMDs was 26 million [1].

Film-makers are exploring the full range of potential subjects for panoramic videos, exploiting the ability of panoramic video to place the viewer at the centre of the action. There is work being done exploring the visual grammar of 360-degree video, such as the impact of cuts on the viewing experience [24] and how allowing users to reorient themselves following cuts can increase the likelihood they will see important content [21]. Motorized swivel chairs have been used to nudge viewers in the direction of important content in 360-degree videos [13]. There are already guidelines on whether directors should make videos that require the user to turn 360 degrees, or whether they should keep the action to approximately 180 degrees to limit the requirement on the user to turn $[14,31]$. It has also been shown in static content that viewers tend to focus on the equator of the viewing sphere [25]. These works appear to indicate that there is a tension between making the scene immersive, and risking that users miss something important. Indeed users sometimes express a fear of missing something in panoramic videos when there is a lot of action [19].

There have been a number of academic investigations on the impact of the physical set-up on the viewing experience of 360-degree video. Generally, these have focused on the display type in use. Fonseca and Kraus explored how the level of immersion of the display impacts the viewing experience of 360-degree videos [11]. In a between-groups experiment, 42 participants watched a 360-degree video about the meat industry. Half of the participants watched the video in an Oculus Rift DK2 HMD, while the remaining participants used a 10.1-inch tablet. The results indicated that the HMD condition significantly enhanced pro-environmental attitude, and increased participants' level of sympathy for the characters in the video.

In a 2017 study by MacQuarrie and Steed, the viewing experience among different virtual reality displays was investigated, including an HMD and a CAVE-like device. In that study, viewers were asked to sit on a fixed chair across all the conditions [17]. In a posthoc discussion it was suspected that the use of a fixed chair may have limited the field of regard (FOR) and thus provided partial guidance for the HMD users about the important viewing directions. Therefore, the HMD viewers' fear of missing out was reduced. In a study by Philpot et al. that compared different displays, the HMD users sat on a swivel chair, while in the CAVE condition a fixed seat was used [22]. Participants in the CAVE-like display mentioned in the interview that the fixed chair encouraged them to look towards the front and discouraged them from turning and exploring. In these studies, chair type seemed to have some influence on how much the users explored the virtual environment, and therefore impacted their viewing experience.

In 2018, a study by Bindman et al. explored the impact of the level of immersion of the viewing device when viewing 360-degree video. They examined the impact of immersion on feelings of presence, narrative engagement and empathy when watching 360-degree media [3]. In their work, 65 participants watched a 360-degree video on either an HMD or a smartphone. Similarly to MacQuarrie and Steed [17], the device type was not found to have an impact on narrative engagement. They also did not find the display had an impact on empathy, but a higher level of immersion did produce stronger levels of self-reported presence.

\subsection{Viewing Experience}

2.2.1 Spatial Awareness. Spatial awareness is a useful indicator for immersive VR experiences. An HMD was found to induce a higher level of spatial awareness compared with a CAVE display, as HMD users significantly preferred natural rotations and could have more efficient navigation [4]. Another study showed that an HMD performed better in enhancing spatial awareness compared with CAVE and TV displays [17].

It has been found that active exploration in the virtual environment can enhance the performance of spatial awareness tasks compared with passive viewing [26, 27]. Another study indicated that physical rotation could be used to understand the virtual positions in panoramic videos: when trying to learn and memorise the routes in the virtual environment, a participant rotated her chair to the turning direction at each crossroad and used physical position against virtual position as a reference of direction [8]. We note that although panoramic videos do not usually afford exploration by movement through the space of the video, the act of turning is a type of exploration, and thus we might expect restrictions on ease of turning to impact spatial awareness.

2.2.2 Incidental Memory. One measure of the utility of panoramic video is a user's memory of the videos. Incidental memory refers to the memories that are unintentionally made during an experience [12]. Participants' memory could be tested through different approaches including free recall, cued recall and recognition [29]. Free recall is to recall elements without any reminders while in cued recall a related concept is provided to assist in memory retrieval. Recognition is to select the elements based on memory using multiple choice questions.

Using immediate free recall tasks, Rizzo et al. identified that the viewers of panoramic videos in an HMD had worse incidental memory compared with those watching videos on a computer monitor [23]. This may have been because the panoramic videos exerted additional cognitive load and distracted viewers from the audio contents. In another study, headset viewers also reported being distracted by exploring the surroundings due to their freedom in viewing [20]. We might thus expect users who can explore more to recall less incidental information.

2.2.3 Concern about Missing Something. A problem of HMDbased videos is the lack of directional control over the users' viewing 
direction. This may lead to participants' fear of missing out, especially when narrative understanding is involved [17]. It has been found that HMD users reported greater concern about whether they were looking in the right direction and whether they missed something, compared with CAVE users [22]. In this study, various types of videos were selected, including those with the characters mainly in the front and those with several characters scattered around, those with narrative elements and those without. Therefore, it was hypothesised that greater concern about missing something would be found for some of the experimental videos from participants with a more restrictive chair.

2.2.4 Engagement. Engagement has been studied in a variety of media. A commonly-used questionnaire is the Measuring Narrative Engagement Questionnaire (MNEQ). This questionnaire contains 12 items that probe four dimensions: narrative understanding, attentional focus, emotional engagement and narrative presence [7]. It is designed for relatively long experiences, whereas panoramic videos are currently shorter, with brief narratives. A framework by Finlay focuses on three aspects: behavioural engagement, cognitive engagement and emotional engagement [10]. Because users in panoramic video can only explore by viewing, in our study we modified this framework to focus on attentional engagement rather than behavioural.

\section{METHODS}

\subsection{Experimental Conditions}

In order to compare the influence of different chairs on users' viewing experience for panoramic videos, this study adopted a between-subject experimental design. The between-subject design was adopted to avoid practice and fatigue effects, as each participant only watched the videos in one of the three conditions:

(1) Fixed condition: The chair seat was fixed and could not swivel.

(2) Half-swivel condition: The chair seat could swivel 180 degrees - 90 degrees to the left and 90 degrees to the right of forward.

(3) Full-swivel condition: The chair seat could swivel completely 360 degrees.
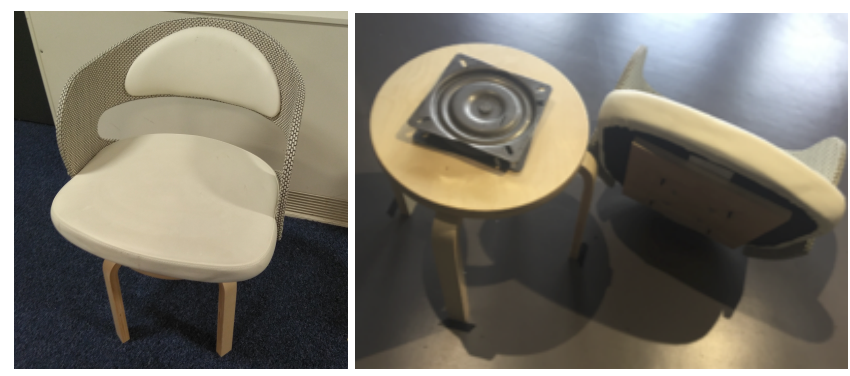

Figure 1: The chair used in the study. On the left, the fully assembled chair as seen by participants. On the right, the disassembled chair showing the swivel mechanism.

The base and seat of the chair were connected by swivel mechanisms, as shown in Figure 1. Two swivel plates were used. One could swivel within 180 degrees and one could swivel fully. In the fixed chair condition, the full swivel plate was fixed by tape to disable the

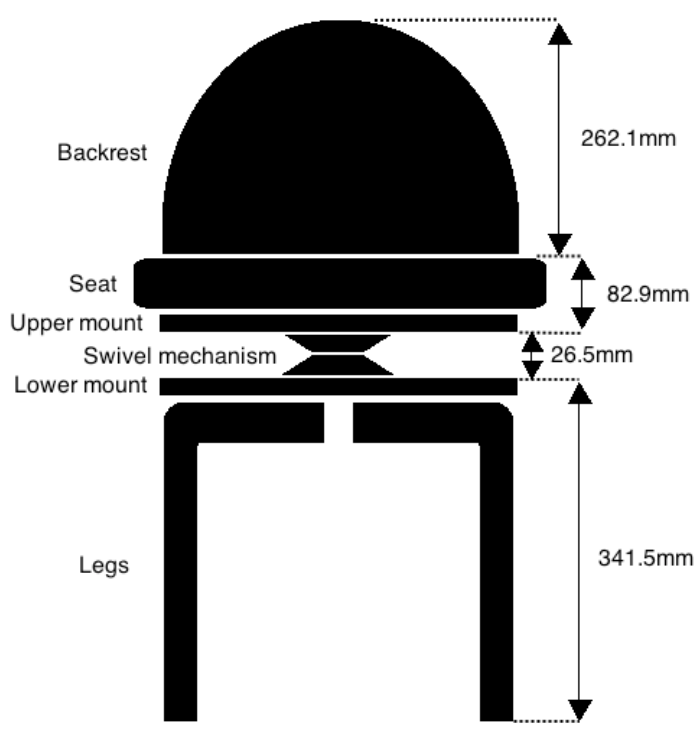

Figure 2: Diagram of chair components.

swivel mechanism. The legs of the chair were shortened by $11 \mathrm{~cm}$ in order to remove the height added by the swivel mechanism and the seat. A diagram showing the heights of the chair components is shown in Figure 2.

\subsection{Hypotheses}

Based on the reviewed literature, this study proposed six hypotheses regarding the viewers' experience.

- H1: With less swivelling chairs (i.e. fixed and half swivel), participants will explore less in the virtual environment.

- H2: With less swivelling chairs, participants will have worse spatial awareness.

- H3: With less swivelling chairs, participants will have better incidental memory.

- H4: With less swivelling chairs, participants will have greater concern about missing something for certain videos.

- H5: With less swivelling chairs, participants will show less engagement.

- H6: We do not expect to find a difference in users' simulator sickness among the three chair conditions.

Note that each of these hypotheses implies sub-hypotheses about the order of the three conditions.

\subsection{Participants}

Participants were recruited through university mailing lists and participant pool websites. A total of 41 participants were recruited in this study, with 19 males and 22 females. The ages ranged from 18 to 38 (mean $=24.8$; standard deviation $=4.59)$. Participants were randomly allocated among the experimental conditions. There were 14 participants in the fixed chair condition, 13 participants in the halfswivel chair condition and 14 participants in the full-swivel chair condition. The session for each participant last for approximately 45-60 minutes. Participants completed a background information 
questionnaire prior to starting that collected data such as age, gender, etc. (for a complete list of all questions and tasks, please see the supplemental material).

\subsection{Apparatus}

This study used the FOVE-DK-0 head-mounded display. The FOVEDK-0 uses an OLED display, with a resolution of 2560x1440 and a refresh rate of $70 \mathrm{~Hz}$. The FOV is 100 degrees. An OSVR infra-red camera was used to correct any IMU drift in orientation tracking. Positional tracking was disabled. The cables of the FOVE were hung through a hanger on the ceiling in order not to restrict the participants from rotating. The software was created using Unity 5.6.1f1 (64 bit). The software ran on a Windows 10 desktop PC with an Intel i7-4790 CPU running at $3.6 \mathrm{GHz}$ with 8GB of RAM, using an NVIDIA GeForce GTX 1080 video card.

\subsection{Stimuli}

In this study, one practice video and five test videos were used as stimuli. All of the videos had a resolution of $4 \mathrm{~K}$. The videos were selected to cover a range of genres, such as narrative content, documentary and dance. Videos were chosen to cover a range of scenarios, such as where the focus of the video takes place in front of the viewer, and where characters move around the viewer to encourage exploration.

Video 1 was a music video. While a central character moves around the viewer, which may encourage exploration, action fills the entire 360 degrees throughout. Video 2 was a narrative piece, in which all important content was contained within the front 180 degrees. Video 3 was a documentary, in which the central character was in the forward direction throughout. Video 4 was a cartoon, in which characters were mainly in the forward direction, but circled around the viewer briefly. Video 5 was a dance video, in which action happened in all directions. For video 5, looking around was essential in order to follow the action of the video. The stimuli are described in more detail, including links to the source videos, in the supplemental material (see Table S1).

These videos were also selected based on their appropriateness for our adopted metrics. For instance, in the video used for testing incidental memory (Video 3), there was some factual, little-known information in the audio. Questions regarding these facts had clear answers and thus could be objectively rated. In the videos aimed at testing spatial awareness (Video 2 and Video 4), the video remained in the same location for a period of time, with target objects located around the environment that could reasonably be remembered.

\subsection{Measures}

3.6.1 Exploration. Eye-tracking data was collected using the FOVE HMD. The distribution of eye gaze was expected to provide insights about users' exploration patterns while watching the videos. The eye tracking data was logged as the coordinates of eye gaze at each time point. The midpoint of the left-eye and right-eye gaze positions was adopted to represent the eye gaze position.

The average rotation of the eyes away from the forward direction was used to measure users' exploration. It was calculated as the average of the absolute differences between the X coordinate of eye gaze at each logged point and the $\mathrm{X}$ coordinate of the original eye

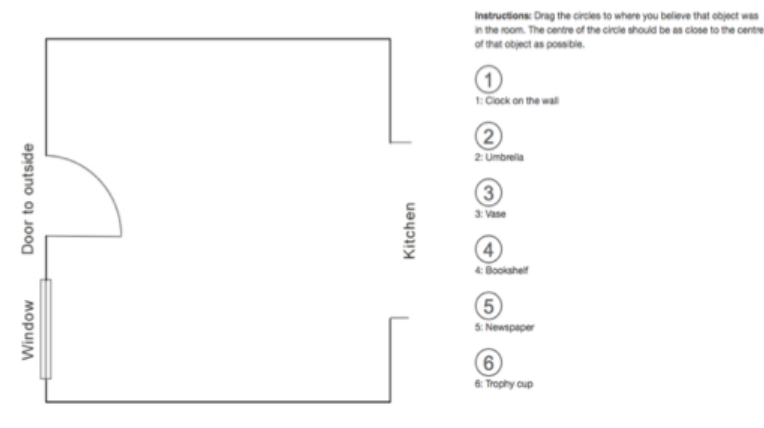

Figure 3: Screenshot of the map placement task for video 4. Instructions read: "Drag the circles to where you believe that object was in the room. The centre of the circle should be as close to the centre of that object as possible."

gaze point. Participants were instructed to start from the forward direction and thus their original eye gaze reflected their forward direction.

3.6.2 Spatial Awareness. Spatial awareness was measured with the use of map placement tasks. The tasks were displayed on a web page, and users asked to drag objects that were present in the scene on to a map of the scene, as shown in Figure 3 (the full details of all questions and tasks are included in supplementary materials). After watching Video 2 and Video 4, participants were asked to drag the circles and duplicate the locations of objects in the virtual environment on a map. The placement tasks had different levels of difficulty, with the selection of objects scattered around the virtual environment as the targets. Participants' performance score was represented as the summed Euclidean distance between the placed location and the ground truth. The scores for the objects that were left unmoved were given the maximum error distance among all the moved objects.

3.6.3 Incidental Memory. Incidental memory was measured with ten questions about the audio content of Video 3. These questions had different levels of difficulty, from straightforward questions to questions that required thought. No cues could be obtained from the visual display. The speakers and the volume of the audio were kept consistent across the three experimental conditions.

The questions asked about factual information and thus had objective answers. As the answer for one question contained three elements (queen/work/drone), correctly answering each one of them would be given $1 / 3$ point. The participants' incidental memory was represented as the numbers of their correct answers. For a full list of questions, see supplemental material (Figure S3).

3.6.4 Concern about Missing Something. Concern about missing something was measured by a five-point Likert scale with two statements: "At times, I was worried I was missing something" "My concern about missing something impacted my enjoyment of this video". The summed score for these two answers was used to represent the overall concern about missing something. This measure was also provided after each video. 
3.6.5 Engagement. Engagement was measured by six questions. These questions were built on three aspects of the viewing experience: attentional engagement, cognitive engagement and emotional engagement, with two questions for each aspect. Example questions include: "I focused my attention on watching these videos" and "I tried my best to understand what was happening in the videos". Participants were asked to answer each item on a five-point Likert scale from "Never" to "Always". Question 6 ("I was absent-minded during watching") was a negatively worded question for measuring attentional engagement and therefore its score was reversed. The total score of the overall engagement was calculated by summing the scores for Question 1-5 and the reversed score for Question 6. For a full list of questions, see supplemental material (Figure S4). The reliability and validity of this questionnaire was examined and factor analysis was conducted to test the dimensions.

3.6.6 Simulator Sickness. This study used the Simulator Sickness Questionnaire (SSQ) as a measure of simulator sickness (SS) [15]. In this study, both pre-exposure and post-exposure SS were measured. The difference between the pre-exposure SSQ score and the post-exposure SSQ score was used to indicate the influence of the virtual reality system.

3.6.7 Semi-Structured Interview. A semi-structured interview was conducted after watching all the videos in order to elicit conversation and gain more insights of users' viewing experience. The interview outline was as follows:

- Feelings

- How do you feel in general about watching the videos?

- Are there any aspects about which you feel satisfied or dissatisfied?

- How do you feel about the viewing experience compared with that of traditional/flat-screen videos?

- Preferences

- Were there any videos that you enjoyed more or less? Why?

- (Further question) Did you enjoy/not enjoy the video(s) because of the content, or other aspects?

- Reasons for rotation and fixation

- I noticed that you frequently/sometimes/seldom swivelled around/turned your head, was there any reason for that? (Based on observation)

- I noticed that you frequently/sometimes/seldom stayed at the fixed position, was there any reason for that? (Based on observation)

These questions provided a general guideline and allowed expansion according to participants' responses. Some of the questions were based on observation during the video-watching session. The interviews were recorded, transcribed and analysed using thematic analysis methodology introduced by Braun and Clarke [5].

\subsection{Procedure}

Before the experiment, participants were allocated to one of the three chair conditions based on randomly-generated integers from one to three. Before the arrival of each participant, if the random number for his/her experimental condition was different from the previous condition, the chair for the previous condition would be disassembled and built again with the according swivel for the upcoming participant.

Upon arrival, participants were asked to sit on the chair. They were then given an information sheet about the experiment and a consent form to fill in. Some points for attention, such as possible simulator sickness, were emphasised. However, the purpose of the study, which was about the effect of the chair, was concealed from them. They were only told that the study was to understand their viewing experience.

Next, participants were asked to complete a pre-study questionnaire about their background information (including gender, age, level of English proficiency, familiarity with 360 video, interest in 360 video and pre-exposure SS) on a laptop. After this, participants were introduced to the experiment. They were instructed not to stand up while watching and were told to inform the researcher if they felt any kind of discomfort. Bottles of water were available in case the participants felt any sickness when using the virtual reality system. During the experiment, participants were instructed to wear the FOVE HMD. They then completed the calibration procedure required for accurate eye tracking in the FOVE HMD. After calibration, participants then watched the practice video to reduce the novelty effect and get familiar with watching panoramic videos with the HMD. Following this, they watched the five experimental videos. After watching each video, participants were told to take off the HMD, and were given a laptop, on which they completed the questions and tasks regarding certain aspects of their viewing experience. Questions for concern about missing something were asked after each video, while map placement tasks were provided after Video 2 and Video 4. The test for incidental memory was presented after Video 3. Participants' eye gaze data was collected by the FOVE HMD for all videos. After watching all the videos, participants were asked to fill in an overall questionnaire about their engagement and post-exposure SS. Semi-structured interviews were then conducted to collect their thoughts and feelings towards the viewing experience. After the experiment, participants were debriefed on the purpose of the study. They were paid $£ 10$ for their participation. This study was approved by the UCL Research Ethics Committee.

\section{RESULTS}

Quantitative data from questionnaires, memory tests, map tasks and eye tracking were analysed with IBM SPSS Statistics 22. As the answers from Likert scales, the scores of the memory test and the map placement tasks were ordinal data, these were analysed using Kruskal-Wallis H Tests. Data from eye tracking was ratio data and thus was analysed with one-way ANOVA. When there was a significant difference among the three conditions, Bonferroni correction was applied for further pairwise comparisons. As there were three comparisons, the significance required for the pairwise comparison was $\mathrm{p}<0.0167$. Qualitative data gained from the semistructured interviews was analysed using thematic analysis.

\subsection{Viewing Experience}

4.1.1 Exploration. As six participants failed in eye gaze calibration and the data for one participant were recorded unsuccessfully in part of the videos, their data were excluded. As a result, eye gaze data from 34 participants was analysed, with 10, 14 and 10 


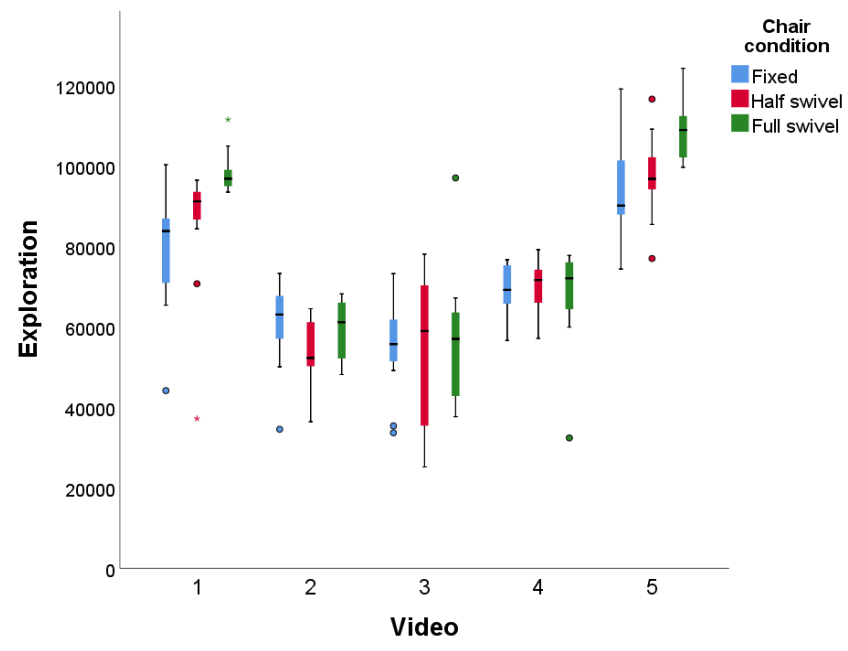

Figure 4: Box plot of exploration for all videos.

participants in fixed, half-swivel and full-swivel chair conditions respectively. The eye gaze for the practice video was also excluded.

Scatter plots of aggregated eye positions (using horizontal and vertical coordinates) provided an overview of the viewers' exploration patterns. For the videos that encouraged exploration (e.g. Video 1), noticeable difference in eye-gaze patterns existed among different chair conditions. It was seen that participants constrained by the chairs focused on the front part, while the eye gaze from those in more swivelling chairs tended to expand their focus of attention across the horizontal axis. For the videos that had contents mainly in the front (e.g. Video 3), the difference across chair conditions was less obvious, as the focus of attention was mainly in the front (scatter plots for these videos are included in the supplemental materials, Figures S5 and S6).

The average rotations (horizontal deviations) of the eyes from forward were then compared across different chair conditions using one-way ANOVA. The result showed that there were significant differences among the three chair conditions in exploration for Video 1 and Video 5, as shown in Table 1. Further Post-Hoc tests (using Bonferroni correction) revealed that the viewers in the fixed chair $(M=79675.9, S D=16000.35)$ significantly explored less for Video 1 than those in the full swivel chair $(M=98577.69, S D=5516.49)$, $\mathrm{p}=0.011<0.0167$. Users in the fixed chair $(\mathrm{M}=92045.17, \mathrm{SD}=11051.52)$ and the half-swivel chair $(M=97205.74, S D=9618.98)$ both significantly explored less for Video 5 than those in the full-swivel chair $(\mathrm{M}=109500.77, \mathrm{SD}=8036.42), \mathrm{p} 1=0.001<0.0167, \mathrm{p} 2=0.013<0.0167$. Figure 4 shows the box plot of exploration for all videos.

This result was partly consistent with Hypothesis 1, which predicted that users in less swivelling chairs would explore less.

4.1.2 Spatial Awareness. The result of Kruskal-Wallis H Test indicated that the difference in spatial awareness between the experimental conditions was significant, $\chi^{2}(2)=6.823, p=0.033<0.05$. The comparisons between each two conditions were examined using Mann-Whitney Test with Bonferroni correction. The result of the pairwise comparisons suggested that participants in the fixed condition (Mean Rank=25.86) produced significantly larger Euclidean
Table 1: Results of One-way ANOVA for exploration across the three chair conditions.

\begin{tabular}{ccc}
\hline Exploration & $\boldsymbol{\chi 2}$ & $\boldsymbol{p}$ \\
\hline Video 1 & 5.148 & $0.012 *$ \\
Video 2 & 1.990 & 0.154 \\
Video 3 & 0.142 & 0.868 \\
Video 4 & 0.300 & 0.743 \\
Video 5 & 8.753 & $0.001 *$ \\
Overall & 2.614 & 0.089 \\
\hline *. p $<0.05$ & &
\end{tabular}

error scores in the map placement task compared with those in the full-swivel condition (Mean Rank=14.43), $\mathrm{p}=0.015<0.0167$. A significant difference was not found for error scores between fixed and half-swivel conditions (Mean Rank=2285), $\mathrm{p}=0.467$, or between half-swivel and full-swivel conditions, $\mathrm{p}=0.058$.

This means that participants in the fixed chair performed significantly worse when testing spatial awareness compared than those in the full-swivel condition. This finding was partly consistent with Hypothesis 2, which proposed that a restrictive chair would reduce the viewers' spatial awareness.

4.1.3 Incidental memory. The Kruskal-Wallis H Test was used to examine the difference in memory scores for all participants among the three chair conditions. The result suggested that there existed no significant difference among the chair conditions, $\chi^{2}(2)=1.173$, $\mathrm{p}=0.556$.

As was suggested by a pilot study, English level might have an influence on participants' performance for our measure of incidental memory. As a result, a background question about their English level was asked. The result showed that there existed a significant difference in the performance scores among participants with different English levels, $\chi^{2}(2)=19.339, p=0.000$. The participants who assessed their English level as intermediate only correctly answered an average of $1.24(\mathrm{SD}=1.05)$ of the 10 questions, while those of advanced level only averaged $2.63(\mathrm{SD}=1.82)$ correct answers. By contrast, participants with superior English level achieved 4.90 $(\mathrm{SD}=2.11)$ correct answers. No participant responded that their English level was novice.

Considering this, a post-hoc analysis was conducted where only participants of superior English level were included. There were 22 filtered viewers, with 7 in fixed condition, 8 in half-swivel condition and 7 in full-swivel condition.

The outcome of a Kruskal-Wallis H Test suggested that the difference in incidental memory among the three conditions reached significance, $\chi^{2}(2)=6.339, p=0.042<0.05$. Further pairwise comparisons indicated that participants in the fixed condition (Mean Rank=15.86) performed significantly better in memory score compared with those in the half-swivel condition (Mean Rank=7.44), $\mathrm{p}=0.014<0.0167$. Figure 5 shows the box plot of performance scores for incidental memory test among different chair conditions, from participants with superior English level.

This result suggested that the chair type may have an impact on incidental memory. However, the result was only significant 


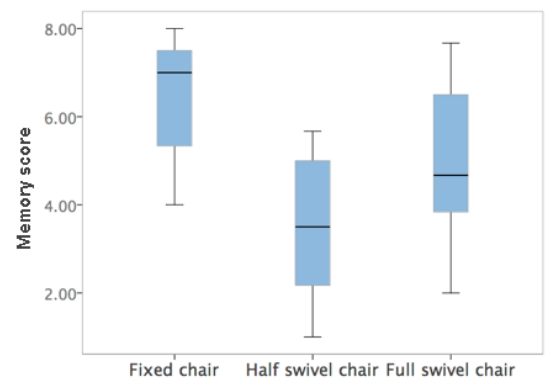

Figure 5: Box plot of performance scores for memory test among participants with different chair conditions.

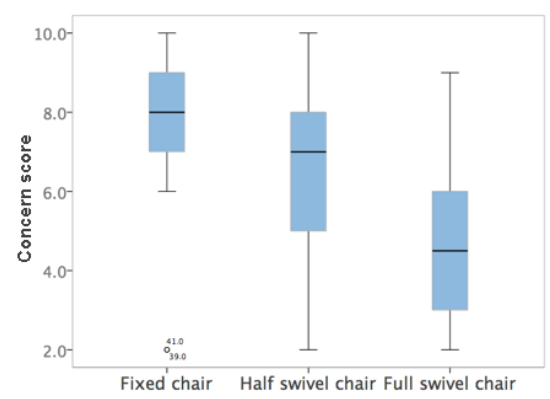

Figure 6: Box plot of concern about missing something for Video 5.

between half-swivel and fixed conditions in a post-hoc analysis. This was not consistent with Hypothesis 3.

4.1.4 Concern about missing something. The data demonstrated a significant difference in concern about missing something for Video $5, \chi^{2}(2)=8.811, p=0.012<0.05$. A complete list of results by video is shown in Table 2. Pairwise comparison with Mann-Whitney Test and Bonferroni correction found that participants sitting on a fixed chair (Mean Rank=27.68) had a significantly higher level of concern about missing something for Video 5 compared with those sitting on the full swivel chair (Mean Rank=14.36), $\mathrm{p}=0.006<0.0167$. Figure 6 provides the box plot of concern about missing something for Video 5. This result partly accorded with Hypothesis 4 .

Table 2: Results of Kruskal-Wallis $H$ Test for concern about missing something across the three conditions.

\begin{tabular}{ccc}
\hline $\begin{array}{c}\text { Concern } \\
\text { about } \\
\text { missing } \\
\text { something }\end{array}$ & $\chi 2$ & Asymp. Sig \\
\hline Video 1 & 0.375 & 0.829 \\
Video 2 & 0.679 & 0.712 \\
Video 3 & 1.535 & 0.464 \\
Video 4 & 1.162 & 0.559 \\
Video 5 & 8.811 & $0.012 *$ \\
Overall & 2.034 & 0.362 \\
\hline$*$. p $\approx 0.05$ & & \\
\hline
\end{tabular}

4.1.5 Engagement. As the questionnaire for viewers' engagement in panoramic videos was created in this study, its reliability and validity were examined. The internal consistency reliability was checked using Cronbach's alpha. The score for question 6 was reversed as it was a negatively worded question. The result of reliability analysis showed that the Cronbach's alpha was 0.601 . Among all the items, the question 4 ("I felt sad when I didn't catch up with the information in the videos") reduced the total reliability, as shown in Table 3. Therefore, this item was deleted. After deleting this item, the Cronbach's alpha became 0.752 and was acceptable. Validity was then tested using Pearson Product Moment Correlations. The outcome suggested that almost all of the correlation values between each two items as well as between each item and the total score reached the significant level (for a list of correlations between all items, see Table S2 in the supplemental materials). Therefore, the validity of this questionnaire was also acceptable.

Table 3: Internal consistency reliability test for engagement questionnaire.

\begin{tabular}{cc}
\hline $\begin{array}{c}\text { Items in } \\
\text { Questionnaire for } \\
\text { Engagement }\end{array}$ & $\begin{array}{c}\text { Cronbach's Alpha if } \\
\text { Item Deleted }\end{array}$ \\
\hline EngageQ1 & .507 \\
EngageQ2 & .511 \\
EngageQ3 & .469 \\
EngageQ4 & .752 \\
EngageQ5 & .493 \\
Reserved EngageQ6 & .552 \\
\hline
\end{tabular}

Factor analysis was conducted to test the dimensions of the questionnaire. The result indicated that there were only existed two principal components, as shown in Table 4, and they could together account for $72 \%$ of the total variance. The first component was made of question 1 ("I enjoyed watching the videos"), question 3 ("I focused my attention on watching the videos"), question 5 ("I tried my best to understand what was happening in the videos") and question 6 ("I was absent-minded during watching"). The second component only contained question 2 ("I learnt something from the videos"). Considering the features of these questions, component 1 was named as "Absorption" while component 2 was named as "Acquisition". The score for "Absorption" was calculated as the sum of the scores for question 1, 3, 5 and the reversed score for question 6, while the score for "Acquisition" was simply the score for question 2. The score for general engagement was the summed score of the two components. Analysis with Kruskal-Wallis H Test suggested that no difference in engagement existed between the three conditions. The box plot of this result is shown in Figure 7. This result was inconsistent with Hypothesis 5, as it was predicted that the chair would influence users' engagement levels.

4.1.6 Simulator sickness. Wilcoxon Test was used to compare the difference between the pre-exposure SSQ score and the postexposure SSQ score, through no significant difference was found between these responses $(p=0.211)$. This suggested that the experiment and the viewing experience were safe for participants. 
Table 4: Factor analysis - Rotated Component Matrix ${ }^{a}$.

\begin{tabular}{|c|c|c|c|c|}
\hline & \multicolumn{2}{|c|}{ Raw } & \multicolumn{2}{|c|}{ Rescaled } \\
\hline & \multicolumn{2}{|c|}{ Component } & \multicolumn{2}{|c|}{ Component } \\
\hline & 1 & 2 & 1 & 2 \\
\hline EngageQ6 & -.681 & & -.849 & \\
\hline EngageQ5 & .621 & & .834 & \\
\hline EngageQ3 & .442 & & .691 & \\
\hline EngageQ1 & .540 & & .675 & \\
\hline EngageQ2 & & 1.167 & & .983 \\
\hline
\end{tabular}

Extraction Method: Principal Component Analysis.

a. Rotation converged in 3 iterations.

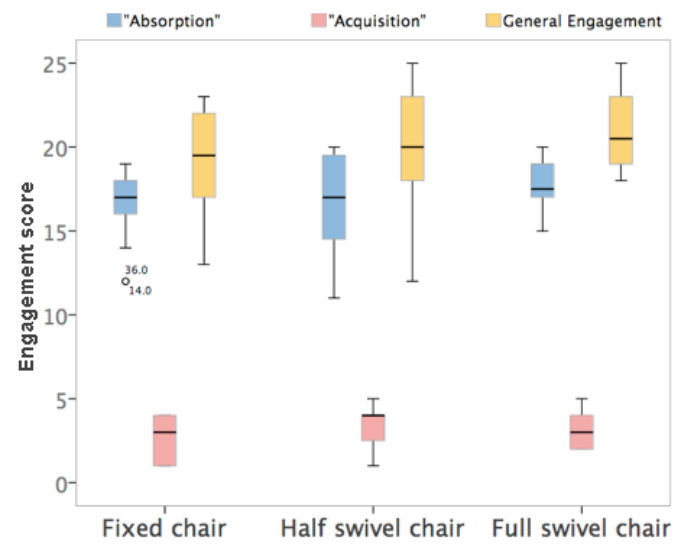

Figure 7: Box plot of engagement among the chair conditions.

The Kruskal-Wallis H Test was used to examine the difference between pre- and post-exposure SSQ scores (changed SSQ) among the three chair conditions. The result suggested that there existed no significant difference among the chair conditions in terms of the changed SSQ, $\chi^{2}(2)=0.045, p=0.978$. This is consistent with Hypothesis 6.

\subsection{Semi-Structured Interview}

Interviews were analysed using thematic analysis to capture patterns of the viewing experience from interview transcripts. In order to identify the differences between experimental conditions, information related to the impact of the chairs was given particular attention. Through thematic analysis, four themes were established: physical discomfort, difficulty following moving objects, reduced orientation, and guided attention.

4.2.1 Physical discomfort. Most of the participants in the fixed chair condition and half-swivel chair condition reported that it was uncomfortable to turn their bodies or heads. This discouraged them from exploring more of the videos.
"It's kind of annoying to twist my back. It isn't comfortable when twisting around." (P6; fixed condition)

"It required too much effort to turn around. I didn't want to move. It's not healthy for my neck." (P18; half-swivel condition)

"It was uncomfortable when I tried to look at the very behind and even to looked at the other side [sic]. So I didn't do much of that." (P30; half-swivel condition)

4.2.2 Difficulty following moving objects. Participants in the fixed and half-swivel conditions found it difficult to follow the movements of the characters in the videos, especially when the characters moved quickly or frequently.

"Because this chair cannot go, like, in a full circle. Sometimes I need to turn this way and sometimes I need to turn that way. The dancers keep moving. I just fail to follow." (P3; half-swivel condition)

"I cannot really turn around and look behind me because of the chair. It's, like, annoying? Especially when the main focus moved behind. I cannot really follow the action." (P12; fixed condition)

This difficulty in following the movement of characters increased their concern about missing something, especially for Video 1, 4 and 5, which involved the main characters moving around. Among them, Video 5 involved the main characters performing in different directions at the same time and it was most frequently mentioned, especially by those in the fixed and half-swivel chair conditions.

"It (Video 5) was annoying because they were consistently moving around, like, really really quickly. And I did definitely miss things with that." (P6; Fixed-chair condition)

"It (the chair) was a bit annoying and also made me miss a bit of details [sic]. This was especially apparent when I'm doing the nom one (Video 4)." (P8; fixed condition)

For the videos in which the main characters were mainly to the front and side, this concern was reduced.

"The bees, it's mainly that guy there, so I'm not really missing that much. But in the cartoon, the baby jumped around and I found I needed to try to quickly turn the chair." (P2; half-swivel condition)

"It's mainly in the side (Video 2). So you weren't concerned there is anything behind you." (P32; half-swivel condition)

4.2.3 Reduced orientation. It was identified that participants' turning around helped them to memorise the locations of the objects. For example, P31 (full-swivel condition) mentioned that "It (the object in the map placement task) should be here... I remember I turned to this side and it was here." Therefore, viewers who rotated more might have better spatial awareness because of this. By contrast, those in more restrictive chairs lacked this assistance, and could therefore have reduced orientation.

4.2.4 Guided attention. Participants, especially those in the fixed and half-swivel conditions, focused mainly on the front part. As the chair guided them to look forward, they seemed to feel they were being given a direction when surrounded by too much information.

"There is too much information. I don't know where to look at, so I just look at the front." (P7; fixed condition).

"Someone popped out here and that guy popped out there. I don't know. They wanted me to explore. But they are way too much [sic]? I just focused on the central part." (P11; fixed condition). 
Some of the viewers believed that the front part could provide most of the important information, so there was "no need to turn around or look behind” (P21; half-swivel condition). Similarly, P35 (half-swivel condition) believed "everything is going to happen in front of you."

\section{DISCUSSION}

\subsection{Fear of Missing Out}

The analysis of eye-tracking data suggested that a restrictive chair discouraged exploration for certain videos. Specifically, participants with a fixed chair explored significantly less for Video 1 than those in the full-swivel chair condition, whereas viewers with a fixed chair and a half-swivel chair both explored significantly less for Video 5 compared with those in a full-swivel chair. This was partly consistent with the hypothesis 1 . While Video 1 and Video 5 both involved main characters moving around, the viewers in restrictive chairs felt physically uncomfortable and experienced difficulty following the movements of the characters, as indicated in the interviews, and thus explored less. While a fixed chair discouraged rotation for both Video 1 and Video 5, the half-swivel chair only significantly restricted the exploration for Video 5 . This might be because in Video 1, there was one main character moving around to lead users' attention, so users were encouraged to follow. By contrast, in Video 5, there were several main characters. When the users in restrictive chairs found it less easy to follow and there was not a single character to guide attention, they may have given up exploring the environment.

Moreover, participants in a fixed chair also suffered from a greater concern about missing something for Video 5. It was assumed by a previous study that a fixed chair might provide guidance to the users and thus reduce their concern about missing out when using HMDs [17]. While this could be true, the videos in their study had the main characters mainly in the forward direction, representing a different scenario from the one discussed here.

A notable point was that participants in a fixed chair had greater concern about missing something than those in a full swivel chair for Video 5, but not for Video 1, although these two videos both involved the characters moving in the background. There are some potential explanations. Firstly, there was one main character in Video 1 who led viewers' attention, whereas there were two or three main focuses in Video 5. As a result, viewers in all of the conditions may have had a greater fear of missing out for Video 5 . This might be because when facing several choices about the focus of attention, the users in the full swivel condition could just choose one and follow it. By contrast, in the fixed chair condition, the chair implicitly guided the users to look forward. While different characters moved to the front, they reminded the fixed-chair users that there were different choices for focus of attention. Thus, these viewers were not free to choose and follow one focus. Another possible explanation was that while there were almost no narrative elements in Video 1, Video 5 conveyed an emotional love story through dancing which involved narrative understanding. As it has been suggested that for videos that require narrative understanding, participants may feel more concern about missing out [17]. Viewers in the fixed chair felt it was harder to follow the movements (indicated during the interviews), and thus that it was harder to follow the narrative. Therefore, it may be that concern about missing out for this video was further enhanced by its narrative elements.

These findings indicate that there is a complex interplay of factors that may need to be considered. These factors include the capabilities of the display, the physical setup such as the chair type, and properties of the video such as genre and character movement. This may be an interesting area for further exploration, in particular how specific properties of the video impact the experience.

\subsection{Spatial Awareness}

It was found that participants in the fixed condition performed worse in terms of spatial awareness compared with those in fullswivel chair condition. This may have been caused by the restriction of the fixed chair, as it discouraged participants from exploring the environment and obtaining a spatial sense of the locations from physical movements, as was indicated in a previous study [16] and in the interviews. This could also have been because the objects tested in the map placement tasks were scattered around the virtual environment, with some of them to the sides and back of the viewer, and participants may have failed to notice these objects. Considering this, when designing panoramic videos, if there is a requirement for spatial orientation or to convey spatial information, producers should consider the at-home viewers' reduced spatial awareness, and think about how to support their spatial recognition or orientation.

\subsection{Incidental Memory}

For participants across all English language levels, the chair condition was not found to impact memory scores. In a post-hoc analysis, it was found that participants in the fixed condition had better incidental memory than those in the half-swivel condition after excluding the effect of English level. It might be that the fixed chair encouraged users to focus on the audio information while the halfswivel chair encouraged more visual exploration and thus distracted the users from the audio. This finding is consistent with a previous study which suggested that participants' memory performances may be poorer for panoramic videos than for videos viewed on a flat screen, possibly because the panoramic videos added cognitive load and distracted users from memorising the audio [23].

However, a concern with this finding is that only the participants who assessed their English language level as superior were included in the post-hoc analysis of incidental memory. As a result, there were only 7-8 participants for each condition, making this result less convincing. Excluding participants with disadvantageous levels of English seemed to be important, however, as the influence of English level on memory test score was highly significant $(\mathrm{p}=0.000)$. As some participants mentioned in the interviews, the man's voice in the video for testing memory was not clear. This perhaps made it harder for participants with weaker English capacities to understand the audio information.

Another concern with this finding is that the results do not show a clear relationship between the restrictive quality of the chair and memory score, with the half-swivel chair performing worst of the three conditions. As noted in [17], memory scores are highly variable between individuals, so these post-hoc results should be treated with caution. 


\subsection{Engagement}

Viewers' engagement was measured by a self-made questionnaire. The questionnaire was found to possess acceptable reliability and validity after removing one of the items. However, factor analysis suggested there were only two components. The first component might be described as "Absorption" as it seemed to focus on the viewers' being absorbed in the videos and engaging with their emotion, attention and cognition. The second component could be named "Acquisition", because it was about obtaining information.

This division was inconsistent with the theoretical framework on which the questionnaire was built. However, as this framework was originally built to study engagement, this inconsistency may be understandable. The current division of "Acquisition" and "Absorption" seems to describe the passive viewing experience better. "Absorption" is a lower level of engagement by which the viewers apply attentional, emotional and cognitive effort when watching a video. By contrast, "Acquisition" is a higher level of engagement by which the viewers not only apply cognitive effort but also succeed in acquiring information. This finding might reveal a possible framework that may suit the passive viewing experience for measuring general engagement. However, as testing a questionnaire would require a large number of participants, the number of participants involved in this study is not large enough to make these results convincing.

\subsection{Interviews}

By conducting semi-structured interviews and analysing the scripts with thematic analysis, four themes about the restrictive chair conditions were extracted: physical discomfort, difficulty following moving objects, reduced orientation, and guided attention.

Viewers sitting in the fixed and half-swivel chairs felt it was uncomfortable to turn their head or twist their back. This seemed to explain why they explored less, especially for the videos that had information around or behind them.

The constrained chairs also made it harder to follow the movements of characters, causing them to feel more worried about missing the information in the videos. However, this concern did not appear when the main focus of the video was in the forward direction. This might explain why participants in restrictive chairs experienced significantly greater concern about missing out than participants in the full-swivel chair for Video 5 (which involved a large amount of character movement) but not for the other videos.

Participants in restrictive chairs found it harder to obtain spatial information, possibly because they did not physically move to gain additional information and/or they failed to notice objects in different parts of the virtual environment. Assistance from physical movements to obtain virtual orientation seem to be consistent with Dalvandi's experiment, in which a participant used physical position against virtual position as a reference of direction [8].

Moreover, viewers were seemingly guided by the chairs to the forward direction when they did not know where to look. They also believed that the forward direction would provide most of the important information. The guided attention from the fixed chair was also suggested by the interviews in a previous study in which the participants mentioned that the non-swivel seat encouraged them to look to the front [22].

\section{CONCLUSION}

This study explored the effect of the chair type on HMD users' viewing experience for 360-degree videos. Three types of chair were built, with different swivelling capacities. The key findings in this study were as follows.

(1) Participants in fixed and half-swivel conditions explored less for certain videos compared with those in the full-swivel condition.

(2) Participants in the fixed condition had worse spatial awareness than those in the full-swivel condition.

(3) In a post-hoc analysis, participants with superior English language abilities in the fixed condition had better incidental memory than those in the half-swivel condition.

(4) Participants in the fixed condition had greater concern about missing something for certain video than those in the full-swivel condition.

(5) No significant difference was found in terms of engagement among the three chair conditions.

(6) No significant difference was found in terms of simulator sickness among the three chair conditions.

(7) Through semi-structured interviews and thematic analysis, four themes were established about the restrictive chairs: physical discomfort, difficulty following moving objects, reduced orientation, and guided attention.

However, it should be noted that this study had some limitations. Firstly, the numbers of participants in each condition was not large and this made the results less convincing, especially for the analysis of incidental memory and our self-made questionnaire on engagement. It may also be for this reason that significant results were only found for several pairs rather than a consistent difference between all of the three conditions. Moreover, as the viewing experience depended largely on the contents of the videos, generalisability of the findings may be limited. In addition, the apparatus used in this study (FOVE HMD) was different from the previous study (Oculus Rift [17]), making the results less comparable.

Future work could examine the effect of viewing condition with larger numbers of participants. Further studies could focus on specific elements, comparing similar videos with and without these elements in order to control these variables in a stricter manner. It is important to note the type of video and the structure of the action, and the impact these aspects seems to have on user behaviour. Overall we believe that the results presented here can provide useful guidance, both on the potential impact of chair type, but also on how to evaluate different viewing experiences.

\section{ACKNOWLEDGMENTS}

This work was supported in part by grant EP/G037159/1 for University College London's Virtual Environments, Imaging \& Visualisation Doctoral Training Centre (http://engdveiv.ucl.ac.uk/) and grant EP/N509577/1 from the UK Engineering and Physical Sciences Research Council (EPSRC).

\section{REFERENCES}

[1] ARtillry. 2018. Data Point of the Week: VR Installed Base to Reach 67 Million by 2021. http://artillry.co/2018/03/26/data-point-of-the-week-vr-installed-base-toreach-67m-by-2021/ [Online; accessed 12-Aug-2018]. 
[2] Brent Ayrey and Christopher Wong. 2017. Introducing Facebook 360 For Gear VR. https://newsroom.fb.com/news/2017/03/introducing-facebook-360-for-gearvr/ [Online; accessed 21-November-2017].

[3] Samantha W Bindman, Lisa M Castaneda, Mike Scanlon, and Anna Cechony. 2018. Am I a Bunny?: The Impact of High and Low Immersion Platforms and Viewers' Perceptions of Role on Presence, Narrative Engagement, and Empathy during an Animated 360Âr Video. In Proceedings of the 2018 CHI Conference on Human Factors in Computing Systems. ACM, 457.

[4] Doug A Bowman, Ameya Datey, Young Sam Ryu, Umer Farooq, and Omar Vasnaik. 2002. Empirical comparison of human behavior and performance with different display devices for virtual environments. In Proceedings of the human factors and ergonomics society annual meeting, Vol. 46. SAGE Publications Sage CA: Los Angeles, CA, 2134-2138.

[5] Virginia Braun and Victoria Clarke. 2006. Using thematic analysis in psychology. Qualitative research in psychology 3, 2 (2006), 77-101.

[6] Dominic Brennan. 2017. Samsung Gear VR Install Base Has Passed 5 Million Headsets. https://www.roadtovr.com/samsung-sold-5-million-gear-vr-headsets/ [Online; accessed 21-November-2017].

[7] Rick Busselle and Helena Bilandzic. 2009. Measuring narrative engagement. Media Psychology 12, 4 (2009), 321-347.

[8] Arefe Dalvandi. 2010. Acquisition of directional knowledge in virtual environments created by panoramic videos. Ph.D. Dissertation. Communication, Art \& Technology: School of Interactive Arts and Technology.

[9] Facebook. 2016. New Steps Toward the Future of Virtual Reality. http://newsroom.fb.com/news/2016/02/new-steps-toward-the-future-ofvirtual-reality/ [Online; accessed 17-November-2017].

[10] Krystina A Finlay. 2006. Quantifying school engagement: Research report. National Center for School Engagement. Denver, CO (2006).

[11] Diana Fonseca and Martin Kraus. 2016. A comparison of head-mounted and hand-held displays for 360 videos with focus on attitude and behavior change. In Proceedings of the 20th International Academic Mindtrek Conference. ACM, 287-296.

[12] Elizabeth Louise Glisky. 2011. Incidental memory. In Encyclopedia of Clinical Neuropsychology. Springer, 1303-1304.

[13] Jan Gugenheimer, Dennis Wolf, Gabriel Haas, Sebastian Krebs, and Enrico Rukzio 2016. Swivrchair: A motorized swivel chair to nudge users' orientation for 360 degree storytelling in virtual reality. In Proceedings of the 2016 CHI Conference on Human Factors in Computing Systems. ACM, 1996-2000.

[14] Jaunt. 2017. Jaunt Field Guide. https://www.jauntvr.com/cdn/uploads/jaunt-vrfield-guide.pdf [Online; accessed 11-Aug-2018].

[15] Robert S Kennedy, Norman E Lane, Kevin S Berbaum, and Michael G Lilienthal. 1993. Simulator sickness questionnaire: An enhanced method for quantifying simulator sickness. The international journal of aviation psychology 3, 3 (1993), 203-220.

[16] Jesper Kjeldskov. 2001. Interaction: Full and partial immersive virtual reality displays. IRIS24 (2001), 587-600.

[17] Andrew MacQuarrie and Anthony Steed. 2017. Cinematic virtual reality: Evaluating the effect of display type on the viewing experience for panoramic video. In Virtual Reality (VR), 2017 IEEE. IEEE, 45-54.

[18] New York Times. [n. d.]. The Daily 360. https://www.nytimes.com/video/thedaily-360 [Online; accessed 17-November-2017].

[19] Katy Newton and Karin Soukup. [n. d.]. The Storyteller's Guide to the Virtual Reality Audience. https://medium.com/stanford-d-school/ the-storyteller-s-guide-to-the-virtual-reality-audience-19e92da57497. Accessed: 13-Aug-2018.

[20] Peter J Passmore, Maxine Glancy, Adam Philpot, Amelia Roscoe, Andrew Wood and Bob Fields. 2016. Effects of viewing condition on user experience of panoramic video. (2016).

[21] Amy Pavel, Björn Hartmann, and Maneesh Agrawala. 2017. Shot orientation controls for interactive cinematography with 360 video. In Proceedings of the 30th Annual ACM Symposium on User Interface Software and Technology. ACM 289-297.

[22] Adam Philpot, Maxine Glancy, Peter J Passmore, Andrew Wood, and Bob Fields 2017. User experience of panoramic video in CAVE-like and head mounted display Viewing Conditions. In Proceedings of the 2017 ACM International Conference on Interactive Experiences for TV and Online Video. ACM, 65-75.

[23] A Rizzo, L Pryor, R Matheis, M Schultheis, K Ghahremani, and A Sey. 2004. Memory assessment using graphics-based and panoramic video virtual environments. In Proc. 5th Intl Conf. Disability, Virtual Reality \& Assoc. Tech.

[24] Ana Serrano, Vincent Sitzmann, Jaime Ruiz-Borau, Gordon Wetzstein, Diego Gutierrez, and Belen Masia. 2017. Movie editing and cognitive event segmentation in virtual reality video. ACM Transactions on Graphics (TOG) 36, 4 (2017), 47.

[25] Vincent Sitzmann, Ana Serrano, Amy Pavel, Maneesh Agrawala, Diego Gutierrez, Belen Masia, and Gordon Wetzstein. 2018. Saliency in VR: How do people explore virtual environments? IEEE transactions on visualization and computer graphics 24, 4 (2018), 1633-1642.
[26] Grégory Wallet, Hélène Sauzéon, Jérôme Rodrigues, and Bernard N’Kaoua. 2008. Use of virtual reality for spatial knowledge transfer: Effects of passive/active exploration mode in simple and complex routes for three different recall tasks. In Proceedings of the 2008 ACM symposium on Virtual reality software and technology. ACM, 175-178.

[27] Grégory Wallet, Hélène Sauzéon, Jérôme Rodrigues, and Bernard NâĂŹKaoua. 2009. Transfer of spatial knowledge from a virtual environment to reality: Impact of route complexity and subjectâÁźs strategy on the exploration mode. Fournal of Virtual Reality and Broadcasting 6, 4 (2009), 572-574.

[28] WAREABLE. [n. d.]. The best Samsung Gear VR apps: Games, videos and experiences to download first. https://www.wareable.com/vr/best-samsung-gear-vrapps-the-games-demos-and-experiences-to-download-first-816 [Online; accessed 20-November-2017].

[29] David Wechsler et al. 1997. Wechsler memory scale (WMS-III). Psychological Corporation San Antonio, TX.

[30] YouTube Creator Blog. 2017. Hot and Cold: Heatmaps in VR. https://youtubecreators.googleblog.com/2017/06/hot-and-cold-heatmaps-in-vr.html [Online; accessed 11-Aug-2018].

[31] YouTube Official Blog. 2017. The world as you see it with VR180. https://youtube.googleblog.com/2017/06/the-world-as-you-see-it-withvr180.html [Online; accessed 11-Aug-2018] 